\title{
AUTOMATIC AND THRESHOLD-FREE EVALUATION OF 3D BUILDING ROOF RECONSTRUCTION TECHNIQUES
}

\author{
Mohammad Awrangjeb \\ Gippsland School of Information Technology \\ Monash University, Vic 3842, Australia \\ E-mail: mohammad.awrangjeb@monash.edu
}

\author{
Clive S. Fraser \\ CRC for Spatial Information \\ University of Melbourne, Vic 3010, Australia \\ E-mail: c.fraser@unimelb.edu.au
}

\begin{abstract}
Although a large number of building roof reconstruction techniques from the remotely sensed data have been proposed, there is a serious lack in standard and automatic evaluation system. The manual evaluation systems are based on human judgment and so may be biased. Moreover, the application of such an evaluation system on a large data set requires a considerable amount of human involvement. In addition, the threshold-based evaluation systems that employ one or more thresholds while making correspondences between the detection and reference sets are also subjective since there is no unique way to choose the thresholds. This paper proposes an automatic and threshold-free evaluation system using the centre distances between the detected and reference entities. It neither requires any human involvement nor uses any thresholds. As a result, the proposed evaluation system can be exploited for a bias free evaluation of any roof reconstruction techniques, even on large data sets.
\end{abstract}

Index Terms - Roof reconstruction, extraction, automatic, threshold-free, evaluation.

\section{INTRODUCTION}

Roof reconstruction implies the extraction of 3D roof information, which includes corners, edges and planes of the roofs from remotely sensed data such as aerial and satellite imagery and digital surface models (DSMs). Although a large number of roof reconstruction techniques have been proposed in the literature [1], a commonly accepted and widely used evaluation system is lacking. In fact, different authors evaluate their techniques using different evaluation systems and metrics. Therefore, it is difficult to compare the performance of different roof reconstruction techniques.

There are many issues related to the current evaluation systems. First, many evaluation schemes [2] do not involve any objective criteria for their reconstructed roof planes. Instead visual results for a limited number of buildings are used for quality assessment. Second, there are also manual evaluation systems [3] that are based on human judgement as to whether the roofs are partially or fully reconstructed or not. These manual evaluation systems are too subjective and therefore the results may be biased. They are also expensive when applied to large data sets. Third, evaluation systems [4] that involve one or more thresholds to decide whether a plane is correctly reconstructed may be biased. Again, the use of thresholds may not work well in all cases. Fourth, there are many evaluation methods $[3,5]$ which show results based on the number of buildings, not on the number of planes. Such results do not reflect the complete scenario of the reconstructed roof planes. Last but not the least, none of the evaluation systems to date consider the area (number of pixels) of the reconstructed roof planes. Since over- and undersegmentations frequently occur in algorithms applied to LIDAR and image data, it is common not to obtain the exact plane boundary. An area or pixel-based evaluation can reflect this scenario.

This paper proposes an automatic and threshold-free evaluation system that does not involve any human judgement or threshold setting to decide whether a plane is correctly reconstructed. The approach considers both object- and pixelbased evaluations. In addition to completeness, correctness and quality metrics, it involves detection and reference crosslap rates, miss factor and branching factor in order to reflect the cases of over- and under-segmentations. The pixel-based evaluation also indirectly reflects the geometric accuracy. The proposed evaluation system has been applied on a previous roof extraction technique [6,7].

\section{EVALUATION SYSTEM}

The proposed evaluation system assumes the roofs are represented by polyhedral models. An individual roof consists of a set of flat planes. Therefore, a roof boundary or a plane can be represented as a 3D polygon having a set of corner points. If a practical roof boundary or plane has a curved side that side can be reasonably approximated by a set of points.

Let $R_{d}$ and $R_{r}$ be the sets of detected and reference roof models, where each individual roof is represented by its (outside) boundary polygon. Furthermore, let $P_{d}$ and $P_{r}$ be the 


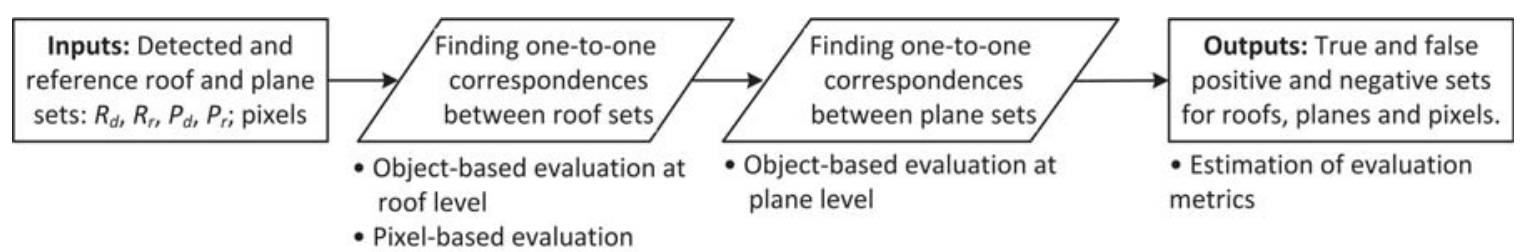

Fig. 1. Proposed evaluation procedure.

sets of detected and reference roof planes, where each individual plane is also represented by a polygon. For each reference plane $p_{r} \in P_{r}$ there is a reference roof ID $i_{r}$ that refers to the corresponding roof in $R_{r}$. So, all reference planes having the same $i_{r}$ consist of a cluster of planes belonging to the same roof.

It is important to find the group of detected planes that belong to the same building. Each detected plane $p_{d} \in P_{d}$ can be represented by a set of (raw or regularised) LIDAR boundary points of the corresponding segmented roof plane. Awrangjeb et al. [6] identifies two detected planes $p_{d 1}$ and $p_{d 2}$ as neighbours if any parts of their boundaries are close (within twice the LIDAR point sampling distance). Consequently, a group of detected planes $P_{1} \subseteq P_{d}$, where a detected plane $p_{d 1} \in P_{1}$ has at least one neighbouring plane $p_{d 2} \in P_{1}$, is considered to be a detected cluster belonging to the same extracted roof $r_{d} \in R_{d}$. As a result, all planes in $P_{1}$ receives the same detected plane ID $i_{d}$. The boundary (polygonal representation) of the roof $r_{d}$ can be easily obtained by considering all planes in $P_{1}$. Note that the finding of detected plane boundaries, neighbourhoods or clusters of detected planes and roof boundaries is performed by the reconstruction technique being evaluated and therefore any involved thresholds are related to the reconstruction technique itself, not to the proposed evaluation system.

The proposed evaluation system, as shown in Fig. 1, works in two steps. First, it is essential to establish one-toone roof correspondences between the roof sets $R_{d}$ and $R_{r}$. One-to-one correspondence means for each entity $r_{d} \in R_{d}$ there will be at most one entity $r_{r} \in R_{r}$ and vice versa. Once a roof correspondence $\left(r_{d}, r_{r}\right)$ is found, then one-to-one plane correspondences between the planes of the corresponding detected and reference clusters $\left(P_{1} \subseteq P_{d}\right.$ and $P_{2} \subseteq P_{r}$ respectively) are established.

In order to find a roof correspondence for an entity $r_{d} \in R_{d}$, the well known point-in-polygon test is followed. Random points inside or along the boundary of $r_{d}$ are generated. The random points may be the LIDAR boundary points or any points inside the plane. Then it is iteratively tested whether at least one of these points resides in any of the reference entity $r_{r} \in R_{r}$. If such an $r_{r}$ is found, then a roof correspondence $\left(r_{d}, r_{r}\right)$ is established and both $r_{d}$ and $r_{r}$ are marked as true positives (TPs). If such an $r_{r}$ is not found, then $r_{d}$ is marked as a false positive (FP). When all the possible correspondences are established for all entities in $R_{d}$, then the entities in $R_{r}$ that do not have corresponding detected planes in $R_{d}$ are marked as false negatives (FNs). These TP, FP and FN statistics are used for object-based evaluation at building or roof level.

Pixel- or area-based evaluation at building level is now carried out. This evaluation can be executed again using the point-in-polygon test. All points in the test area are generated using a regular grid whose resolution is kept fixed at a predefined value, for example, the same value as the input image resolution, LIDAR point sampling distance or a constant $0.25 \mathrm{~m}$. For a roof correspondence $\left(r_{d}, r_{r}\right)$, let the set of pixels which reside inside $r_{d}$ be $A_{d}$ and the set of pixels which reside inside $r_{r}$ be $A_{r}$. Pixels that reside within the overlapping area of $r_{d}$ and $r_{r}$ are true positive pixels (TPX $=A_{d} \cap A_{r}$ ), those which reside in $r_{d}$ but not in $r_{r}$ are false positive pixels $\left(\mathrm{FPX}=A_{d} \backslash A_{r}\right)$ and those those which reside in $r_{r}$ but not in $r_{d}$ are false negative pixels $\left(\mathrm{FNX}=A_{r} \backslash A_{d}\right)$. Once pixels in all the TP roofs are processed, pixels in the roofs that were marked as FNs and FPs, are now directly added to the FNX and FPX sets. Note that since roofs are comprised of planes, the pixel-based evaluation at plane level will be the same as the pixel-based evaluation at building level.

For object-based evaluation at plane level, all the planes in the corresponding plane clusters $P_{1}$ and $P_{2}$, related to a roof correspondence $\left(r_{d}, r_{r}\right)$, are now examined. Let the number of planes in $P_{1}$ and $P_{2}$ be $n_{d}$ and $n_{r}$. Using the pixel sets $A_{d}$ and $A_{r}$, two metrics $M_{d}$ ( size $\left.n_{d} \times n_{r}\right)$ and $M_{r}$ (size $\left.n_{r} \times n_{d}\right)$ are estimated. Further, let the pixels that reside inside a detected plane $p_{d} \in P_{1}$ be $a_{d} \in A_{d}$ and those that reside inside a reference plane $p_{r} \in P_{2}$ be $a_{r} \in A_{r}$. The array element $M_{d}(i, j)$, where $1 \leq i \leq n_{d}$ and $1 \leq j \leq n_{r}$, represents the percentage of detected pixels (from a detected plane $p_{i}$ ) which also reside in a reference plane $p_{j}$. Similarly, The array element $M_{r}(j, i)$, where $1 \leq j \leq n_{r}$ and $1 \leq i \leq n_{d}$, represents the percentage of reference pixels (from a reference plane $p_{j}$ ) which also reside in a detected plane $p_{i}$. Through use of $M_{d}$ a list of detected planes $L_{r}=\left\{p_{d 1}, p_{d 2}, \ldots\right\}$ that maximally overlap a reference plane $p_{r}$ can be obtained. Similarly, use of $M_{r}$ allows a list of reference planes $L_{d}=\left\{p_{r 1}, p_{r 2}, \ldots\right\}$ that are maximally overlapped by a detected plane $p_{d}$ to be obtained. Entities in $L_{r}$ (and $L_{d}$ ) are sorted in descending order of the overlapping percentages with $p_{r}$ (and $p_{d}$ respectively).

In order to find a plane correspondence $\left(p_{d}, p_{r}\right)$, it is firstly checked whether $p_{d}$ is the detected plane that has 
caused the most overlap with $p_{r}$ and vice versa. This means the detected plane that has the maximum value among the planes in $L_{r}$ is $p_{d}=p_{d 1}$ and the reference plane that has the maximum value among the planes in $L_{d}$ is $p_{r}=p_{r 1}$. If the above condition is met, then $\left(p_{d}, p_{r}\right)$ is an eligible plane correspondence and both $p_{d}$ and $p_{r}$ are marked as true positive planes (TPPs). After establishing all the eligible plane correspondences based on the above condition, the following condition based on the first or second most overlapping entities is applied to establish other plane correspondences. For a detected plane $p_{d}$ that does not yet have a corresponding reference plane, both $p_{r 1}$ and $p_{r 2}$ from $L_{d}$ are checked. If $p_{r 1}$ does not yet have a corresponding detected plane, then the two largest overlapping entities $p_{d 1}, p_{d 2} \in L_{r 1}$ are checked. If $p_{d 1}=p_{d}$ or $p_{d 2}=p_{d}$, then $\left(p_{d}, p_{r 1}\right)$ is a new plane correspondence and both $p_{d}$ and $p_{r 1}$ are marked as TPPs. If $p_{r 1}$ already has a correspondence, but $p_{r 2}$ does not, then the two largest overlapping entities $p_{d 1}, p_{d 2} \in L_{r 2}$ are checked. If $p_{d 1}=p_{d}$ or $p_{d 2}=p_{d}$, then $\left(p_{d}, p_{r 2}\right)$ is a new plane correspondence and both $p_{d}$ and $p_{r 2}$ are marked as TPPs. After checking all the detected planes that do not yet have correspondences, a similar check can be executed for all reference planes that also as yet do not have correspondences.

Thereafter, if a detected or a reference plane still does not have a correspondence, it is marked as a false positive plane (FPP) or a false negative plane (FNP). A detection crosslap $C_{d}$ occurs for a detected plane $p_{d}$ if a reference plane $p_{r k} \in L_{d}$, where $k=1,2, \ldots$, still remains without a correspondence in the detected plane set $P_{1}$. Similarly, a reference cross-lap $C_{r}$ occurs for a reference plane $p_{r}$ if a detected plane $p_{d l} \in L_{r}$, where $l=1,2, \ldots$, still remains without a correspondence in the reference plane set $P_{2}$. Finally, all planes in a detected roof $r_{d} \in R_{d}$ that does not have a correspondence in $R_{r}$ are marked as FPPs. Similarly, all planes in a reference roof $r_{r} \in R_{r}$ that does not have a correspondence in $R_{d}$ are marked as FNPs.

\section{DATA SET AND RESULTS}

The test data set employed is from Aitkenvale, a suburban area in Queensland, Australia. Fig. 2 shows the extracted roof planes for this data set (see also [6, 7]).

Two dimensional reference data sets were created by monoscopic image measurement using the Barista software [8]. All visible roof planes larger than $10 \mathrm{~m}^{2}$ were digitized. Since no reference height information was available for the building roof planes, only the planimetric accuracy has been evaluated in this study. Since each of the extracted plane boundaries consists of raw LIDAR points, it can be decided that the accuracy in height completely depends on the input LIDAR data.

Since all the buildings were correctly detected no buildings were wrongly identified, the object-based completeness, correctness and quality at per building level were $100 \%$.

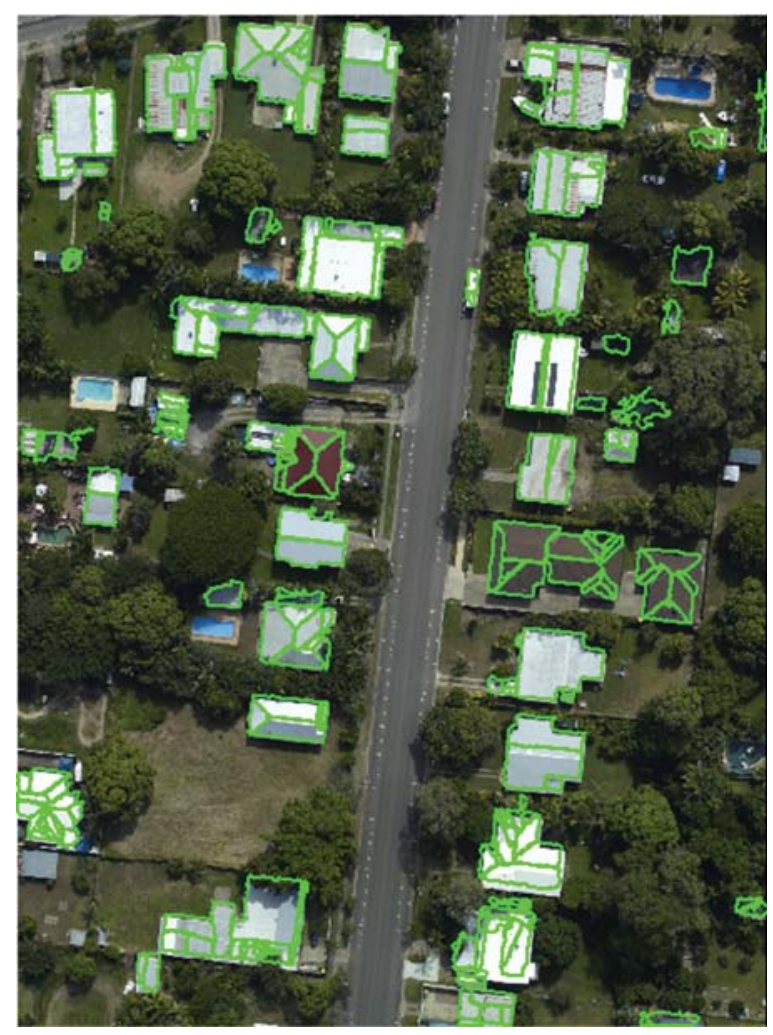

Fig. 2. Extracted roofs on the test data set.

However, since some true planes were missed and some roof planes were wrongly constructed on trees, at plane level the object-based estimations were completeness $98.3 \%$, correctness $99.4 \%$, quality $97.7 \%$, detection cross-lap rate $1.7 \%$ and reference cross-lap rate $27.3 \%$. The pixel-based estimations were completeness $89.9 \%$, correctness $94.2 \%$, quality $85.2 \%$, branching factor $6.1 \%$ and miss factor $11.3 \%$.

Fig. 3 shows some examples of roof extraction on the test data set. Some small roof extensions and detail roof structure were correctly extracted as shown in Figs. 3(a) and (b). Sometimes, partially occluded planes were correctly extracted (Fig. 3(c)). Sometimes, complex structures were correctly extracted as shown in Fig. 3(d). Nevertheless, some false planes were found on the surrounding trees around some buildings (Fig. 3(e)). Moreover, some small garden shades were missed due to occlusion by trees (Fig. 3(f)).

\section{CONCLUSIONS}

The proposed evaluation system is automatic and thresholdfree. It assumes the roofs are represented as polyhedral models. Therefore, the system can be used as a bias-free evaluation system for assessment of other 3D reconstruction techniques that generate polyhedral roof models. In contrast to manual systems that require an extensive human labour, the proposed system can be applied to large data sets without any 

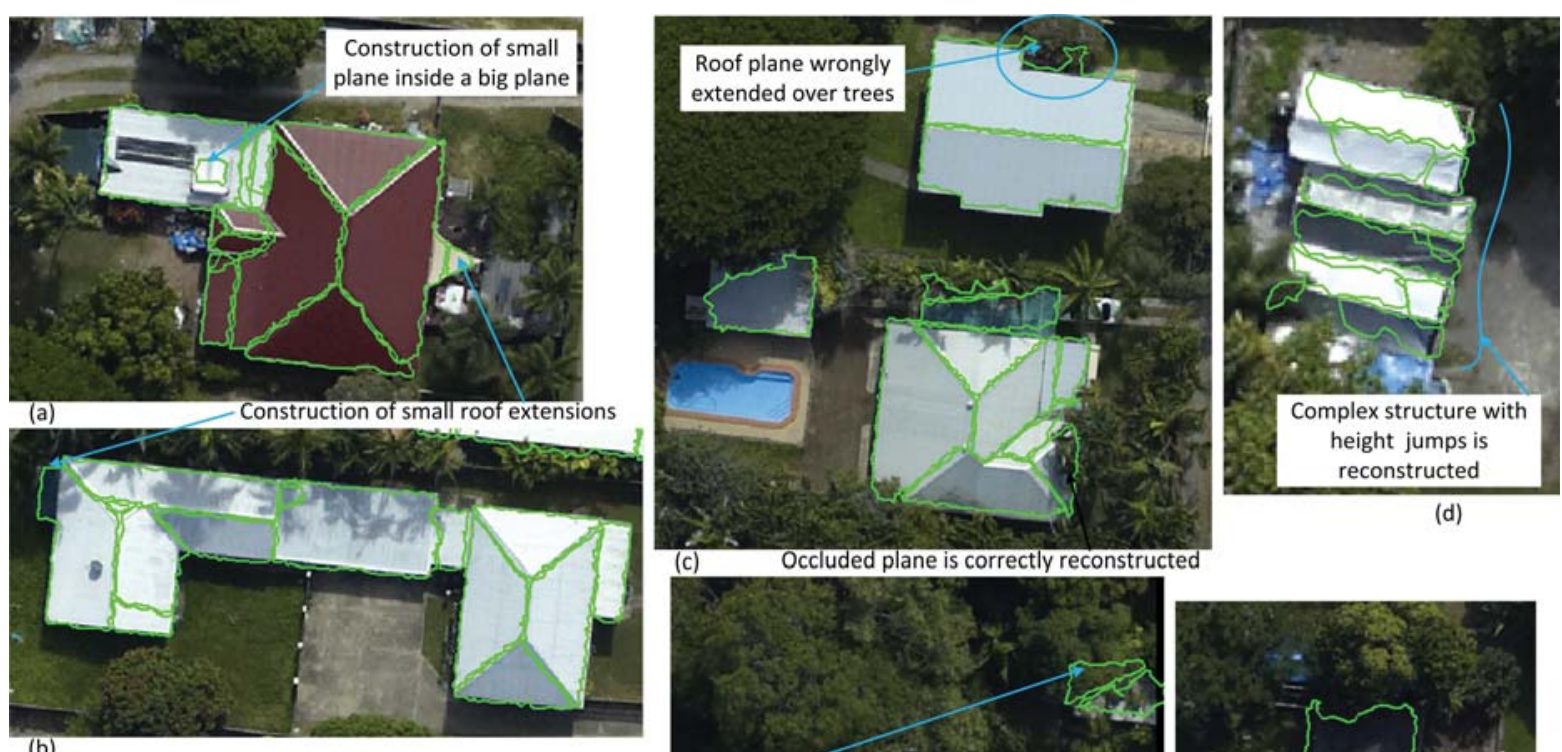

(d)

(b)

False planes due to trees

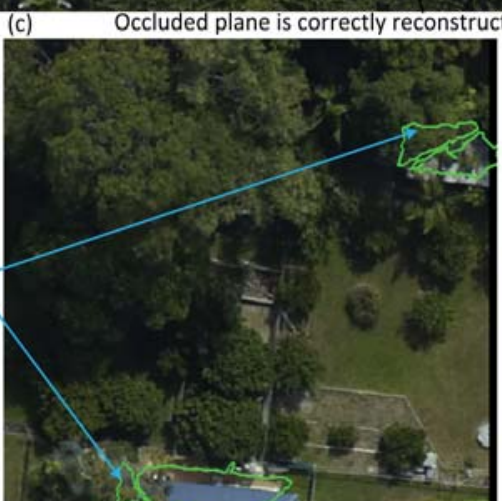

(e)

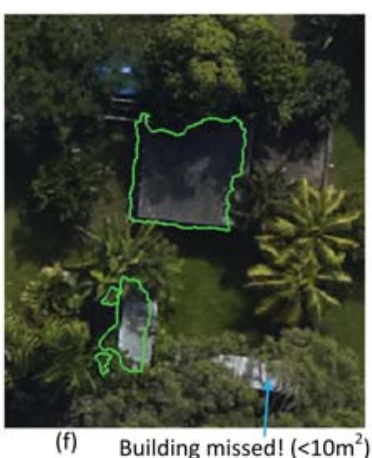

(f) Building missed! $\left(<10 \mathrm{~m}^{2}\right)$

Fig. 3. Examples of roof extractions.

human involvement.

\section{Acknowledgment}

Dr. Awrangjeb is the recipient of the Discovery Early Career Researcher Award by the Australian Research Council (project number DE120101778). The Aitkenvale data set was provided by the Ergon Energy (www.ergon.com.au).

\section{REFERENCES}

[1] N. Haala and M. Kada, "An update on automatic 3d building reconstruction," ISPRS Journal of Photogrammetry and Remote Sensing, vol. 65, no. 6, pp. 570-580, 2010.

[2] J. Park, I. Y. Lee, Y. Choi, and Y. J. Lee, "Automatic extraction of large complex buildings using lidar data and digital maps," International Archives of the Photogrammetry, Remote Sensing and Spatial Information Sciences, vol. XXXVI, no. 3/W4, pp. 148-154, 2006.

[3] L. Chen, T. Teo, C. Hsieh, and J. Rau, "Reconstruction of building models with curvilinear boundaries from laser scanner and aerial imagery," Lecture Notes in Computer Science, vol. 4319, pp. 24-33, 2006.
[4] R. J. You and B. C. Lin, "A quality prediction method for building model reconstruction using lidar data and topographic maps," IEEE Transactions on Geoscience and Remote Sensing, vol. 49, no. 9, pp. 3471-3480, 2011.

[5] L. Cheng, J. Gong, M. Li, and Y. Liu, "3d building model reconstruction from multi-view aerial imagery and lidar data," Photogrammetric Engineering \& Remote Sensing, vol. 77, no. 2, pp. 125-139, 2011.

[6] M. Awrangjeb, C. Zhang, and C. S. Fraser, "Automatic reconstruction of building roofs through integration of lidar and multispectral imagery," in ISPRS Annals of the Photogrammetry, Remote Sensing and Spatial Information Sciences, Melbourne, Australia, 2012, vol. I-3, pp. 203-208.

[7] M. Awrangjeb, C. Zhang, and C. S. Fraser, "Automatic extraction of building roofs using lidar data and multispectral imagery," ISPRS Journal of Photogrammetry and Remote Sensing, vol. DOI: 10.1016/j.isprsjprs.2013.05.006, 2013.

[8] Barista, "The barista software," 2011. 